\author{
International Journal of Health Research \\ and Medico-Legal Practice \\ Open access full-text article \\ Available at www.ijhrmlp.org
}

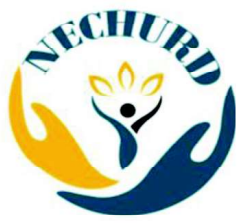

\title{
A clinical, electrophysiological and radiological study of optic neuropathies in Northeast India: hospital-based study
}

\author{
Bawri Satish ${ }^{1}$, \\ Goswami Munindra ${ }^{2}$, \\ Kayal AK', \\ Das Moromi ${ }^{4}$, \\ Das Shubra \\ ${ }^{1}$ Former Senior Resident \\ Mobile: +917896185995 \\ Email: satishbawrighy@gmail.com \\ ${ }^{2}$ Professor \\ ${ }^{3}$ Former Professor and Head \\ ${ }^{4}$ Professor and Head \\ Department of Neurology \\ Gauhati Medical College and Hospital, \\ Guwahati, Assam \\ ${ }^{5}$ Professor \\ Department of Ophthalmology, FAAMC, \\ Barpeta, Assam
}

Received on: Oct 14, 2020

Editorial approval on: Dec 30, 2020

Checked for plagiarism: Yes

Peer review: Double-blinded

Peer review comments: Four

Editor who approved:

Prof. Putul Mahanta
Background and aims: Optic neuropathy refers to disease of the optic nerve which can present in isolation and/or in association with other neurological or systemic diseases. The present study has been undertaken to study the clinical, electrophysiological and radiological features of various optic neuropathies. Materials and methods: This is a prospective observational study and conducted from August 2015 to July 2017 at Gauhati Medical College, Guwahati. Detailed neurological evaluations along with investigation were done in all patients. Results: A Total of 112 patients were included among which 57(50.9\%) were male and $55(49.1 \%)$ were female. Mean age and duration of symptoms was 35.44 years\& $2.06 \pm 0.97$ weeks respectively. Out of 112 patients, bilateral involvement was noted in 57(50.9\%) and unilateral in 55(49.1\%), so, total 169 abnormal eyes and 55 normal eyes were examined. Associated ocular symptoms were ocular pain, colour vision defect, subjective contrast sensitivity and relative afferent pupillary defect. On fundoscopy; patients were grouped into involvement of optic disc (IOOD/Papillitis), retrobulbar and neuroretinitis on the basis of affected part of optic nerve. Relevant positive findings were aquaporin-4 IgG antibody, Angiotensin converting enzyme, Antinuclear Antibody with reactive VDRL, Toxoplasma serology, anti hepatitis $C$ virus, leptospira serology and low serum vitamin $B_{12}$ level. VEP, OCT, CSF and neuro imaging study were done in all patients. Patients were further classified etiologywise among which demyelinating patients' group (MS, NMOSD and AIDON), i.e., $65(58.03 \%)$ was major group. Other etiologies included ischemic, Neurosarcoidosis, toxic and others. Conclusion: The differential diagnosis of optic neuropathy is extensive and apart from demyelination, other causes like ischemic, nutritional, toxic, infective and autoimmune should be considered. All patients need long term follow up.

Keywords: Optic neuropathy; electrophysiological; radiological; etiology.

Cite this article as: Bawri Satish, Goswami Munindra, Kayal AK, Das Moromi, Das Shubra. A clinical, electrophysiological and radiological study of optic neuropathies in Northeast India: hospital-based study. Int J Health Res Medico Leg Prae 2021 JanJun;7(1):25-31. Doi: 10.31741/ijhrmlp.v7.i1.2021.4. 


\section{INTRODUCTION}

Optic neuropathy occurs in a variety of clinical setting and can present in isolation and/or associated with other neurological or systemic diseases. The key features of optic neuropathy are visual loss (affecting visual acuity, colour vision, or visual field) accompanied by abnormal optic disc appearance or relative afferent pupillary defect (RAPD). The most common causes of optic neuropathy are demyelination, ischemic, toxic and autoimmune disorder. In a tropical country like India, apart from the usual suspects, nutritional and infective should also be considered. Multiple Sclerosis (MS) is moderately prevalent in India (Pandit L et al). ${ }^{1}$ Clinical and demographic features are similar to that in the west. Several Indian studies have shown that Optic Neuritis (ON) is the initial manifestation in 23.6 to $53.3 \%$ of patients with Multiple Sclerosis (Syal P et al. ${ }^{2}$, Gangopadhyay G et al.). ${ }^{3}$

The present study has been undertaken to study the clinical, electrophysiological and radiological features of various optic neuropathies.

\section{MATERIAL AND METHODS}

In the present study, all patients irrespective of age and sex who presented with history suggestive of optic neuropathy (history of visual loss affecting visual acuity, colour vision, or visual field accompanied by abnormal optic disc appearance or a relative afferent pupillary defect) were included. The exclusion criteria were (i) patients with altered sensorium, (ii) diagnosed cases of optic atrophy, (iii) intracranial space occupying lesions and (iv) patients with history of trauma. It is a prospective observational study undertaken at Gauhati Medical College, Guwahati and conducted from August, 2015 to July, 2017. Detailed neurological evaluation along with complete blood count (CBC) including erythrocyte sedimentation rate (ESR), liver function test (LFT), renal function test (RFT), electrolytes, Venereal Disease Research Laboratory (VDRL), antinuclear antibody (ANA) and chest X-ray (CXR) done in every patient. Leptospira, Toxoplasma, Angiotensin converting enzyme (ACE) were done in selected patients. Other investigations were done as and when needed as per clinical conditions. Cerebrospinal fluid (CSF) study consisted of total cell count including differential count, sugar, protein, Acid Fast Bacilli (AFB), gram's stain, fungal stain, Adenosine Deaminase (ADA), VDRL, Cryptococcal Antigen, polymerase chain reaction (PCR) for herpes virus, tuberculosis (TB), Japanese encephalitis (JE), ACE, oligoclonal band (OCB). Visual field charting by automated perimetry, optical coherence tomography (OCT), electrophysiological studies include Visual Evoked Potential (VEP) done in all patients. Neuroimaging of brain including orbit and spine was contemplated in all patients. Magnetic Resonance Imaging (MRI) was done in majority of patients although computerized tomography (CT) of brain and orbit was considered in few patients due to financial constrains. Statistical testing was conducted with the statistical package for the social science system version SPSS 17.0.

\section{RESULTS}

Among 112 patients in the study 57(50.9\%) were male and $55(49.1 \%)$ were female. Mean age was 35.44 years (Standard Deviation (SD) of 15.19 and range 16-81 years (Figure 1).

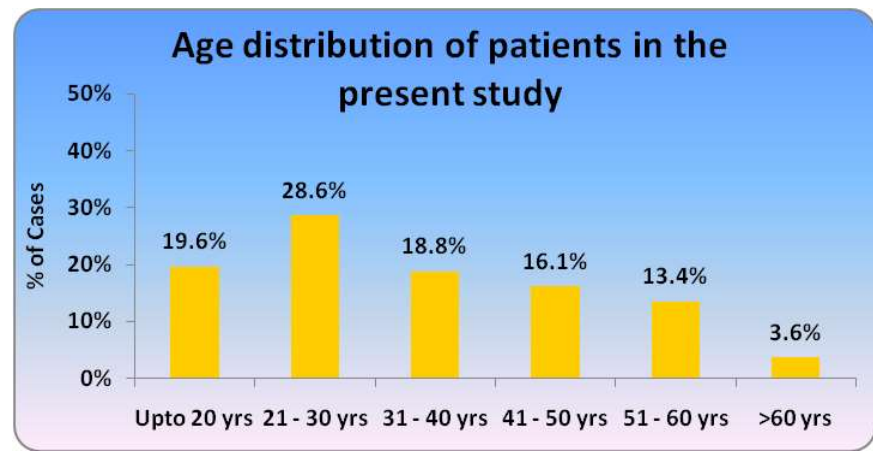

Figure 1 Bar diagram showing age distribution of patients affecting most common in 21-30 years of age group

The mean duration of symptoms was $2.06 \pm 0.97$ weeks (range 0.4-5.4 weeks). Out of 112 patients, bilateral (B/L) involvement was noted in $57(50.9 \%)$ and unilateral (U/L) in $55(49.1 \%)$ of patients. So, total 169 abnormal eyes and 55 normal eyes were examined. At the time of presentation, first attack was noted in $97(86.60 \%)$ of patients, second attack in $14(12.5 \%)$ of which 10 patients were of MS and 4 patients

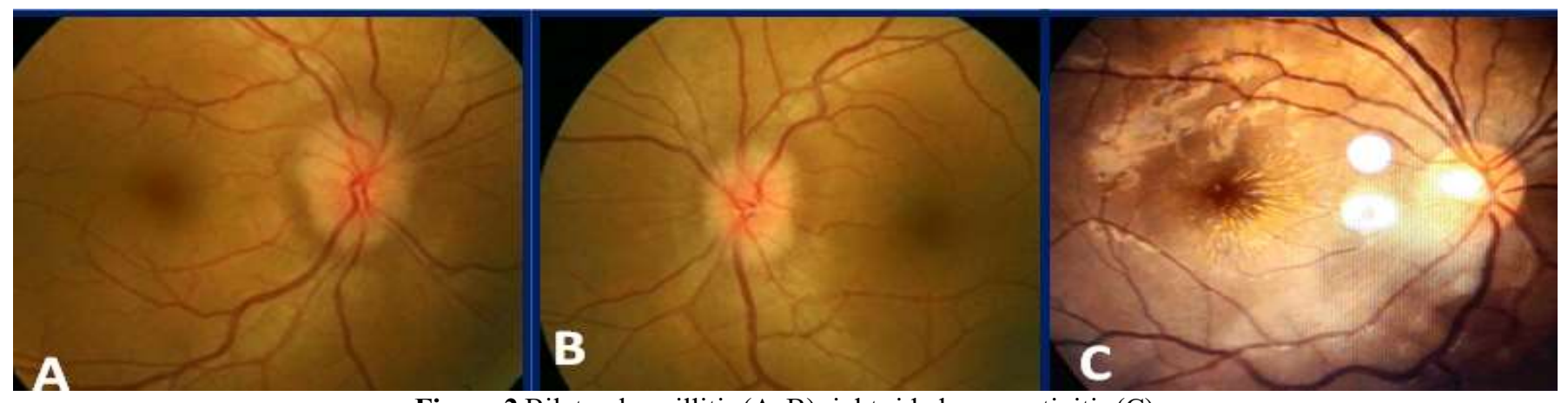

Figure 2 Bilateral papillitis (A, B) right sided neuroretinitis (C) 
were of Neuromyelitis Optica Spectrum Disorder (NMOSD) and third attack noted in $1(0.90 \%)$ patient of NMOSD. Ocular pain was noted in $84(75 \%)$, colour vision defect in $105(93.8 \%)$, subjective contrast sensitivity in $91(81.3 \%)$, redness of eyes in $12(10.7 \%)$ and RAPD in $61(27.23 \%)$ of patients. Out of 224 eyes on fundoscopy, patients grouped into involvement of optic disc (IOOD/Papillitis) in $141(62.9 \%)$, neuroretinitis in $6(2.7 \%)$ and retrobulbar in 22(9.8\%) (Figure 2).

Additional significant neurological features noted in the study were headache in $41(36.6 \%)$, hemiplegia in $6(5.4 \%)$, paraplegia in $4(3.6 \%)$, quadriplegia in $8(7.1 \%)$, sensory findings in $37(33 \%)$, cerebellar involvement in $2(1.8 \%)$, bladder and bowel in 12(10.7\%) and Lhermitte's sign in $4(3.6 \%)$ patients. Out of 112 patients, history of diabetes mellitus and hypertension was elicited in $12(10.7 \%)$ patients, patients on antitubercular therapy (ATT) in $8(7.1 \%)$, chronic kidney disease (CKD) on haemodialysis in $1(0.9 \%)$, chronic liver disease (CLD) in $1(0.9 \%)$ and $1(0.9 \%)$ patient on

Table 1 Relevant abnormal investigation parameters

\begin{tabular}{|l|l|c|}
\hline Investigations & Frequency & Percentage \\
\hline Aquaporin-4 IgG antibody & 14 & $12.5 \%$ \\
\hline ANA including others & 3 & $2.6 \%$ \\
\hline Low serum vitamin B12 & 3 & $2.6 \%$ \\
\hline $\begin{array}{l}\text { Anti hcv including } \\
\text { others parameters }\end{array}$ & 1 & $0.89 \%$ \\
\hline Leptospira serology & 1 & $0.89 \%$ \\
\hline Toxoplasma serology & 2 & $1.78 \%$ \\
\hline Serum VDRL & 3 & $2.6 \%$ \\
\hline Serum ACE & 10 & $8.9 \%$ \\
\hline Screening for LHON & 1 & $0.89 \%$ \\
\hline $\begin{array}{l}\text { Meningeal biopsy } \\
\text { (non-significant) }\end{array}$ & 2 & $1.78 \%$ \\
\hline
\end{tabular}

vinblastine for Non Hodgkin's Lymphoma. Baseline investigations revealed raised ESR in 41(36.60\%), deranged LFT in $10(8.90 \%)$, and deranged RFT in $1(0.89 \%)$ patient. Other relevant investigations were positive aquaporin $4 \mathrm{IgG}$, ACE, ANA including profile, low vitamin $\mathrm{B}_{12}$, VDRL, Toxoplasma serology, anti HCV, Screening for Hereditary Leber's (LHON) and Leptospira (Table 1).

CSF analysis revealed pleocytosis with low to normal sugar and normal to high protein with raised ADA in $7(6.25 \%)$. Other specific positive test in CSF were OCB in 7(6.25\%), ACE in $10(8.9 \%)$, VDRL in $3(2.60 \%)$ of patients. Electrophysiology study (VEP) of 112 patients was recorded among which 169 eyes were abnormal and 55 were normal. Out of 224 eyes, 106(47.32\%) eyes showed prolongation of latency and normal amplitude among which demyelinating group. This group include multiple sclerosis (MS), neuromyelitis Optica Spectrum Disorder (NMOSD) and acute idiopathic demyelinating optic neuritis (AIDON) and this group consisted of $85(80 \%)$ eyes. $24(10.71 \%)$ eyes showed normal latency and decreased amplitude among which ischemic and toxic consisted of 23(95.84\%) of eyes. $39(17.41 \%)$ eyes showed prolongation of latency and decreased amplitude among which inflammatory, toxic, nutritional and demyelinating consisted of 33(84.61\%) of eyes. Visual Field Charting by automated perimetry revealed centrocaecal scotoma in 24(10.71\%), central scotoma in $4(1.8 \%)$, inferior arcuate scotoma in $4(1.8 \%)$, altitudinal in $4(1.8 \%)$, triple quadrant scotoma in $4(1.8 \%)$, double arcuate scotoma in 2(1\%). OCT - Full Macular Thickness (FMT in $\mu$ ), Total Macular Volume (TMV in $\mathrm{mm}^{3}$ ) and Retinal Nerve Fibre Layer (RNFL in $\mu$ ) of 112 patients among which 169 eyes were abnormal and 55 eyes were normal. Eyes (both normal and abnormal) examined on fundoscopy revealed IOOD/Papillitis in 141(62.9\%), neuroretinitis in 6(2.7\%) and $77(34.4 \%)$ were normal of which $22(9.8 \%)$ were retrobulbar and 55(24.6\%) were normal eyes. Findings were statistically analysed and $p$ value $(<0.001)$ is significant for all the grouped analysed. MRI brain including orbit (plain and contrast) was

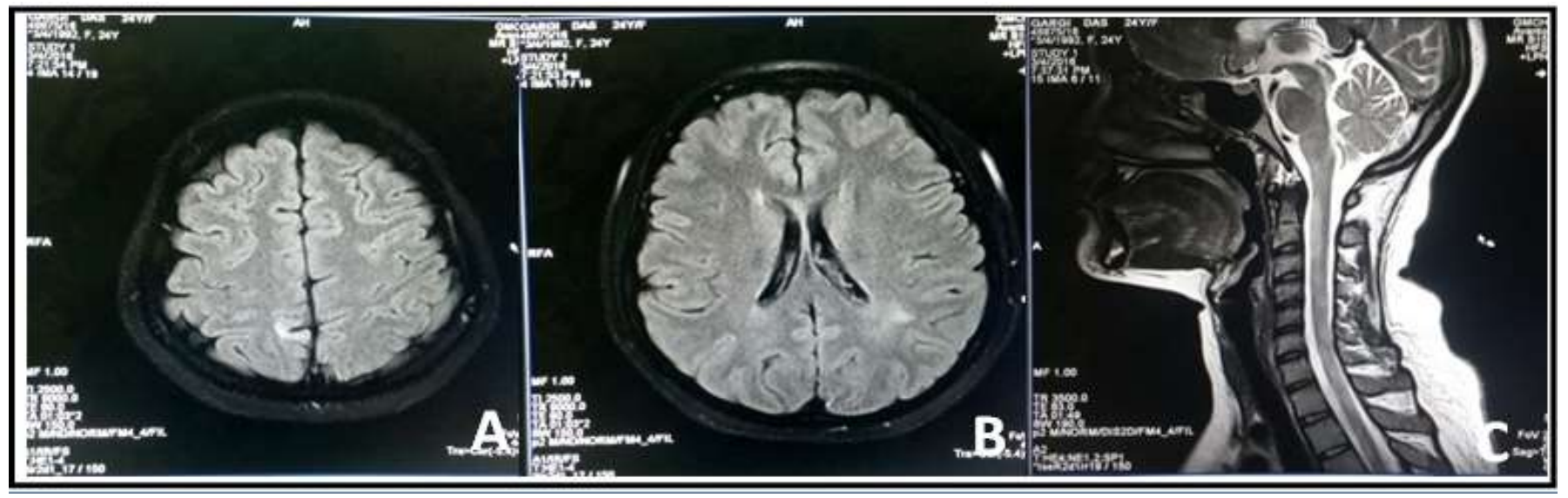

Figure 3 (A, B, C) MRI showing T2 and FLAIR hyper intensities denoting demyelinating lesions in Brain and spinal cord 
done in $76(67.85 \%)$ patients which revealed optic nerve abnormalities (enhancement/enlargement) in 57(75\%) patients, periventricular lesions in $15(19.73 \%$ ) patients of multiple sclerosis, white matter ischemic changes in 14(18.42\%) patients. Out of 30 patients in which MRI Spine was done longitudinal extensive transverse myelitis (LETM) noted in $10(33.33 \%)$ and acute partial transverse myelitis (APTM) in 1(3.33\%). CT scan of brain with orbit (plain and contrast) done in 36(32.14\%) patients which revealed optic nerve abnormalities in $3(8.33 \%)$ patients and others were nonsignificant (Figure 3).

\section{DISCUSSION}

The optic nerve, the extension of the central nervous system, is really a tract and not a peripheral nerve. It begins anatomically at the optic disc, but physiologically and functionally within the ganglion cell layer of retina. The optic nerve is about $50 \mathrm{~mm}$ long and extends from the eye to the optic chiasm. It has four portions: Intraocular or the optic disc $(1 \mathrm{~mm})$, Intraorbital $(25 \mathrm{~mm})$, Intracanalicular $(9 \mathrm{~mm})$ and intracranial portion $(16 \mathrm{~mm})$. Arterial supply is from the ophthalmic artery and the veins draining the optic head and the remaining orbit have no valves and freely anastomose which ultimately drain into the central vein of the retina. Once the diagnosis of optic neuropathy has been made, wide differential diagnosis of possible etiologies like demyelinating, inflammatory, ischemic, toxic others should be considered. If the optic nerve head is swollen on ophthalmoscopy, then the term papillitis or anterior $\mathrm{ON}$ is used and if it is normal on ophthalmoscopy, then it is called retrobulbar ON (Cogan DG et al). ${ }^{4}$

Based on the site of involvement, $\mathrm{ON}$ can be categorized into four main subtypes ( Osborne BJ et al) $)^{5}$

1. Papillitis: involvement of the optic disc.

2. Perineuritis: involves the optic nerve sheath while the optic disc may/may not be swollen.

3. Neuroretinitis: Concomitant swelling of the optic nerve and the macula. Exudates that form around the macula give the appearance of a star.

4. Retrobulbar neuritis: involvement of optic nerve behind the eye.

In the present study of 112 patients, 57 (50.9\%) were male \& $55(49.1 \%)$ were female, mean age of the patients was 35.44 years (SD - $15.19 \&$ range $16-81$ years) \& mean duration of symptoms was $2.06 \pm 0.97$ weeks (range 0.4 5.4 weeks). Comparison with various studies is summarized (Table 2)

Table 2 Various studies conducted with their age and sex distribution

\begin{tabular}{|c|c|c|c|c|c|}
\hline Author & Gangopadhay et al. ${ }^{3}$ & Jain et al. $^{6}$ & Syal et al., ${ }^{2}$ Jena $\mathrm{SS}^{7}$ & Bansil et al. ${ }^{8}$ & Present study \\
\hline Years covered by study & 1989-1999 & 1957-1980 & 1986-1998 & 1990 & 2014-2017 \\
\hline Population & Indian & Indian & Indian & Indian & Indian \\
\hline No of patients & 45 & 354 & 100 & 81 & 112 \\
\hline Mean age & 30.5 & 27 & 28.5 & 27.5 & $34.6 \pm 11.855$ \\
\hline Female:male & $1.50: 1$ & $1.32: 1$ & $1.32: 1$ & $2.25: 1$ & $1.5: 1$ \\
\hline
\end{tabular}

Further 112 patients were classified according to etiology, of which NMOSD consisted of 18(16.07\%) patients; MS consisted of $15(13.39 \%)$ patients, AIDON consisted of $32(28.57 \%)$ patients. Hence if these three groups (MS, NMOSD and AIDON) were taken together, then the total number of patients will be $65(58.03 \%)$ patients were of demyelinating etiology. AIDON is by far the most common type of optic neuritis throughout the world. Although the clinical syndrome of acute optic neuritis has been well recognized for many years, much information about $\mathrm{ON}$ was obtained from the optic neuritis treatment trial (ONTT; Roy $\mathrm{W}$ Beck et al). ${ }^{9}$ Other etiology were ischemic consisted of $12(10.71 \%)$ patients, probable Neurosarcoidosis consisted of $10(8.9 \%)$ patients, toxic consisted of $9(8.03 \%)$ patients, Luetic consisted of $3(2.67 \%)$ patients , nutritional consisted of $3(2.67 \%)$ patients , autoimmune consisted of $3(2.67 \%)$ patients, Toxoplasma consisted of $2(1.78 \%)$ patients, neuroretinitis of possible viral etiology consisted of $2(1.78 \%)$, hepatitis C consisted of $1(0.89 \%)$ patient, Leptospira consisted of $1(0.89 \%)$ patient , hereditary Leber's consisted of $1(0.89 \%)$ patient (Table 3).

In the present study $\mathrm{B} / \mathrm{L}$ involvement was seen in $57(50.9 \%)$ and $\mathrm{U} / \mathrm{L}$ in 55(49.1\%). Further, progression (PR), associated ocular pain, colour vision defect and subjective contrast sensitivity were noted in which 94(83.92\%) patients' symptoms were non progressive (NP). Bilateral involvement was more common in patients of demyelinating etiology. Among 32 patients of AIDON, 2 patients developed features of MS on follow up. In other etiologies like Ischemic, Neurosarcoidosis and toxic, patients also had predominantly bilateral involvement. Adie et al., ${ }^{10}$ found only one case of B/ $\mathrm{L} \mathrm{ON}$ in series of 70 patients. In retrospective analysis by Morrissey et al., ${ }^{11}$ in 23 adults found that causes of $\mathrm{B} / \mathrm{L}$ 
Table 3 Etiology wise patients' distribution

\begin{tabular}{|c|cc|ccc|}
\hline Etiology & Bilateral & Unilateral & Total & Avg. age (years) & Sex(m/f) \\
\hline NMOSD & 10 & 8 & 18 & $32.33 \pm 15.537$ & $6 / 12$ \\
\hline MS & 5 & 10 & 15 & $34.6 \pm 11.855$ & $6 / 9$ \\
\hline AIDON & 14 & 18 & 32 & $28.59 \pm 11.12$ & $17 / 15$ \\
Ischemic & 7 & 5 & 12 & $52.67 \pm 14.49$ & $4 / 8$ \\
Neurosarcoidosis & 6 & 4 & 10 & $40.7 \pm 12.597$ & $5 / 5$ \\
\hline Toxic & 8 & 1 & 9 & $34.67 \pm 11.53$ & $7 / 2$ \\
\hline Luetic & 1 & 2 & 3 & $45 \pm 21.79$ & $3 / 0$ \\
\hline Nutritional & 1 & 2 & 3 & $39 \pm 20.66$ & $3 / 0$ \\
\hline Autoimmune & 3 & 0 & 3 & $41.67 \pm 7.638$ & $1 / 2$ \\
\hline Toxoplasma & 0 & 2 & 2 & $23 \pm 7.07$ & $1 / 1$ \\
\hline Neuroretinitis & 0 & 2 & 2 & $21.5 \pm 2.12$ & $2 / 0$ \\
\hline Hepatitis C & 1 & 0 & 1 & 33 & $1 / 0$ \\
\hline Leptospira & 0 & 1 & 1 & 81 & $0 / 1$ \\
\hline Hereditary Leber's & 1 & 0 & 1 & 22 & $1 / 0$ \\
\hline Total & 57 & 55 & 112 & & $59 / 53$ \\
\hline
\end{tabular}

simultaneous acute ON seen in $5(22 \%)$ who developed clinical evidence of MS. In many of these series, the time interval between attacks in the two eyes is not stated. Park K et al., ${ }^{12}$ observed Uhthoff's phenomenon in $48.1 \%$ of their patients. Jena SS et al., ${ }^{7}$ in their study found Uhthoff's phenomenon in 5 patients $(3.2 \%)$ out of 157 patients whereas in the present study Uhthoff's phenomenon is noted in $4(3.6 \%)$ patients. Ischemic optic neuropathies (IONs; Hayreh SS et al.) ${ }^{13}$ constitute a major cause of blindness or seriously impaired vision among the middle aged and elderly population. IONs consist primarily of two types: anterior ischemic optic neuropathy (AION) and posterior ischemic optic neuropathy
(PION). The term 'autoimmune optic neuritis' has been suggested for cases of optic neuritis in which there is both serologic evidence of vasculitis, such as positive ANA, but no signs of systemic involvement other than the optic neuropathy; and progressive visual loss that tends to be responsive to treatment with systemic corticosteroids and that is often steroid dependent. Moreover, in the present study including probable Neurosarcoidosis patients didn't suggest pulmonary involvement both clinically and radiologically. In the present study, Neurosarcoidosis was categorized as per zajicek diagnostic criteria (Ferriby D et al.) ${ }^{14}$ (Table 4).

Table 4 Zajicek diagnostic criteria

\section{Definite}

1. Histologic confirmation of affected tissue

\section{Probable}

\section{Evidence of CNS inflammation on MRI or CSF}

2. Evidence of systemic sarcoidosis with histologic confirmation and/or at least two of the following indirect indicators: FDG-PET, gallium scan, chest imaging, serum ACE

\section{Possible}

1. Above criteria not met

The optic neuropathy in tubercular meningitis (TBM) in $8(7.1 \%)$ patients was due to ATT in the studied patient as it occurred after 7-10 days of starting ATT and was not due to other causes of visual loss in TBM as supported by repeat investigation including neuroimaging which didn't showed any new feature. Ethambutol is undoubtedly most often implicated in toxic optic neuropathy. The potential for visual impairment was recognized soon after it was introduced.
Optic neuritis due to Isoniazid has been described sporadically among adult patients. The onset of visual symptoms generally occurs within ten days of starting antitubercular therapy but may occur even two to three months after initiation of therapy.

Analysis of initial perimetry in the ONTT (Roy W. Beck et al $)^{9}$ showed that the most common presenting pattern was a diffuse field defect (48\%), with altitudinal/arcuate defects in $20 \%$, and central/cecocentral loss in only $8 \%$ and it has long 
been postulated that $\mathrm{ON}$ tends to affect the papillomacular bundle with resultant central/cecocentral scotoma in $\mathrm{ON}$ patients. While studying CSF of ON patients, Puccioni SM et al. ${ }^{15}$ concluded CSF OCB in $85 \%$ of patients with definite MS. Further studies have showed that patients with positive OCB have a worse prognosis in comparison to those with negative bands, and the risk of progression to a second attack is doubled. In the analysis of VEP, Kiiski HS et al., ${ }^{16}$ showed P100 latencies to be prolonged in MS compared to healthy controls. In the study by Halliday et al., ${ }^{17}$ and Matthews et al., ${ }^{18} \mathrm{VEP}$ was prolonged and abnormal in almost all patients of ON and stated VEP was more sensitive than MRI in respect to ON. As per ONTT (Roy W Beck et al), ${ }^{10}$ the VEP is often abnormal in patients with ON and an abnormal VEP in a clinically diagnosed $\mathrm{ON}$ does not alter the diagnostic or treatment. In terms of OCT, Z Habot-Wilner et al., ${ }^{19}$ demonstrated flattening of the foveal contour, thickening of the neurosensory retina, and accumulation of subretinal fluid in all studied eyes. A vast change was seen in the line of optic neuropathies with the widespread availability of MRI leading to CT scan role to a little. As per ONTT (Roy W Beck et al.), ${ }^{9}$ periventricular white matter signal abnormalities on MRI consistent with MS had been reported in 40 to $70 \%$ of cases of isolated ON. MRI with gadolinium may show enhancing lesions in 26 to $37 \%$ of patients with isolated ON and may increase the detection of disease activity. MRI generally cannot distinguish acute demyelinating optic neuritis from other forms of inflammatory optic neuropathy. e.g., sarcoidosis, syphilis (Cornblath WT et al.). ${ }^{20}$ Patients having prior nonspecific neurologic symptoms with previous history of optic neuritis with abnormal MRI scan having three or more lesions and increased CSF oligoclonal bands have high risk for developing MS. Comparison of MRI findings between NMOSD and MS are summarized (Table 5).

Table 5 Comparison of MRI findings between NMOSD and MS

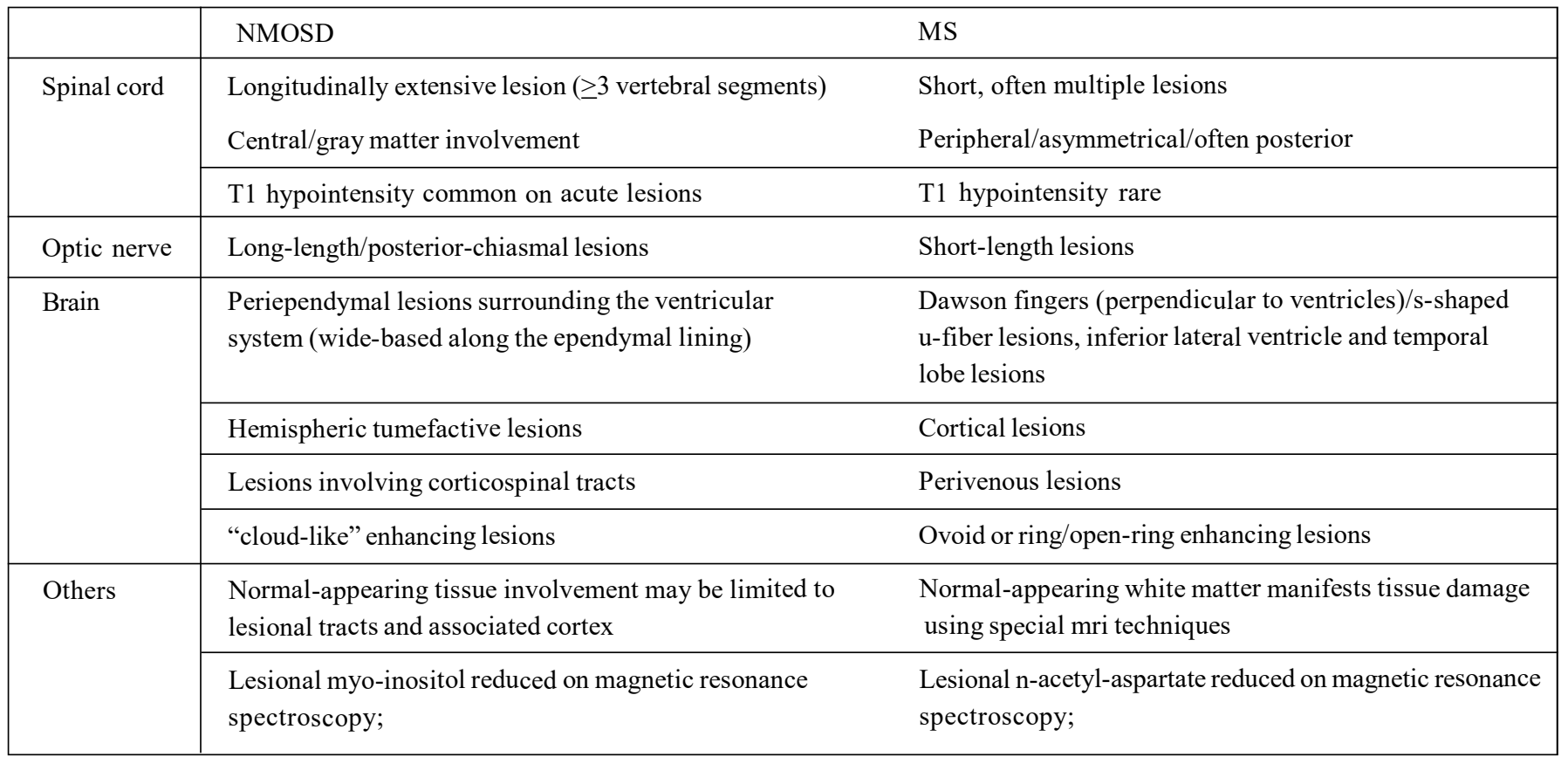

\section{CONCLUSION}

Our study showed that optic neuropathy can have varied etiology. Though demyelinating disease was the major group but other causes of optic neuropathies like ischemic, nutritional, toxic, infective and autoimmune should be considered. A proper diagnosis can only lead to optimal management and follow up.

Ethical clearance: Taken.

Conflict of interest: Nil.

Source of funding: None declared.

\section{REFERENCES}

1. Pandit L, Kundapur R. Prevalence and patterns of demyelinating central nervous system disorders in urban Mangalore, South India. Mult Scler 2014 Oct;20(12):1651-3.

2. Syal P, Prabhakar S, Thussu A, Sehgal S, Khandelwal N. Clinical profile of multiple sclerosis in north-west India. Neurol India 1999 Jan 1;47(1):12.

3. Gangopadhyay G, Das SK, Sarda P, Saha SP, Gangopadhyay P, Roy TN. Clinical profile of multiple sclerosis in Bengal. Neurol India1999 Jan 1;47(1):18.

4. Cogan DG. Optic Nerve: Lesions. Neurology of the 
Visual System. Springfield: Charles C. Thomas; 1980:150-180.

5. Osborne BJ, Volpe NJ. Optic neuritis and risk of MS: differential diagnosis and management. Cleve Clin J Med 2009 Mar 1;76(3):181-90.

6. Jain S, Maheshwari MC. Multiple sclerosis: Indian experience in the last thirty years. Neuroepidemiology 1985 Nov 5;4(2):96-107.

7. Jena SS, Alexander M, Aaron S, Mathew V, Thomas MM, Patil AK et al. Natural history of multiple sclerosis from the Indian perspective: experience from a tertiary care hospital. Neurol India 2015 Nov 1;63(6):866.

8. Bansil S, Singhal BS, Ahuja GK, Ladiwala U, Behari M, Friede R. Comparison between multiple sclerosis in India and the United States- a case-control study. NeurologyAmerican academy of neurology $1996 \mathrm{Feb} 1 ; 46(2): 385$ 7.

9. Roy W. Beck. Optic neuritis study group. The clinical profile of optic neuritis: experience of the optic neuritis treatment trial. Arch Ophthalmol 1991 Dec 1;109(12):1673.

10. Adie WJ. Observations on the etiology and symptomatology of disseminated sclerosis. $\mathrm{Br} \mathrm{J}$ Ophthalmol 1932 Dec 3;2(3752):997.

11. Morrissey SP, Borruat FX, Miller DH, Moseley IF, Sweeney MG, Govan GG, et al. Bilateral simultaneous optic neuropathy in adults: clinical, imaging, serological, and genetic studies. J Neurol Neurosurg Psychiatry Res 1995 Jan 1;58(1):70-4.

12. Park K, Tanaka K, Tanaka M. Uhthoff's phenomenon in multiple sclerosis and neuromyelitis optica. Eur $\mathrm{J}$ Neurol 2014 Aug 28;72(3-4):153-6

13. Hayreh SS. Acute ischemic disorders of the optic nerve: pathogenesis, clinical manifestations and management. Ophthalmol Clin North Am 1996;9:407-42.

14. Ferriby D, De Seze J, Stojkovic T, Hachulla E, Wallaert B, Blond S, et al. Manifestations cliniques et approche therapeutique de la neurosarcoïdose: 40 cas. Rev Neurol (Paris) 2000;156(11):965-75.

15. Polman CH, Reingold SC, Banwell B, et al. Diagnostic criteria for multiple sclerosis: 2010 revisions to the McDonald criteria. Ann Neurol 2011;69:292-302.

16. Lukes SA, Crooks LE, Aminoff MJ, Kaufman L, Panitch HS, Mills C. Nuclear magnetic resonance imaging in multiple sclerosis. Ann Neurol 1983 Jun 1;13(6):592601.

17. Halliday AM, McDonald WI, Mushin J. Delayed visual evoked response in optic neuritis. The Lancet 1972 May 6;1(7758):982-5.

18. Matthews WB, Small DG, Small MA, Pountney E. Pattern reversal evoked visual potential in the diagnosis of multiple sclerosis. J Neurol Neurosurg Psychiatry Res 1977 Oct 1;40(10):1009-14.

19. Habot-Wilner Z, Zur D, Goldstein M, Neudorfer M. Macular findings on optical coherence tomography in cat-scratch disease neuroretinitis. Eye (Lond) 2011 Aug; 25(8):1064-68.

20. Cornblath WT, Quint DJ. MRI of optic nerve enlargement in optic neuritis Neurology- American academy of neurology 1997 Apr 1;48(4):821-5. 